\title{
A146 B CELL RECEPTOR REPERTOIRE ANALYSIS IN CLINICALLY INVOLVED AND UNINVOLVED SKIN OF SYSTEMIC SCLEROSIS PATIENTS TREATED WITH CD2O DEPLETION THERAPY: BASELINE AND FOLLOW-UP
}

Stefano Alivernini, ${ }^{1,2}$ Paul L Klarenbeek, ${ }^{2}$ Silvia L Bosello, ${ }^{1}$ Marieke E Doorenspleet, ${ }^{2}$ Barbera D van Schaik, ${ }^{3}$ Rebecca E Esveldt, ${ }^{2}$ Antoine $\mathrm{H}$ van Kampen, ${ }^{3}$ Frank Baas, ${ }^{4}$ Paul P Tak, ${ }^{2}$ Gianfranco Ferraccioli, ${ }^{1}$ Niek de Vries ${ }^{2}$ Division of Rheumatology, Catholic University of the Sacred Heart, Rome, Italy; ${ }^{2}$ Clinical Immunology and Rheumatology Department, Academic Medical Center, Amsterdam, The Netherlands; ${ }^{3}$ Department of Clinical Epidemiology, Biostatistics and Bioinformatics, Academic Medical Center, Amsterdam, The Netherlands; ${ }^{4}$ Department of Genome Analysis, Academic Medical Center, Amsterdam, The Netherlands

10.1136/ard.2010.149005.13

Background B cells may play an important role in the pathogenesis of systemic sclerosis (SSc), as suggested by reports in the effectiveness of rituximab (RTX). To find support for a role of clonal B cell activation in SSc we studied the presence of clonal $\mathrm{B}$ cell expansions in the involved skin using newly developed next generation sequencing protocols, which provide quantitative data on up to 1000000 clones in one run.

Objective investigate whether expanded $\mathrm{B}$ cell clones are present in involved and uninvolved skin of SSc patients, and how they are influenced by RTX therapy.

Methods mRNA was isolated from involved and uninvolved skin samples of 3 SSc patients (with active diffuse disease) taken before and after 12 months after RTX-therapy. A linear amplification with multiplex primers for all V(ariable)families of the heavy-chain (BCR) was performed. The amplified products contain the CDR3 of the BCRs of all cells which can be used as 'clonal fingerprint' for each cell. The samples were analysed using a Genome Sequencer FLX (454). The frequency of each clone was determined by its CDR3 frequency ( $\%$ of all CDR3s analysed). Clones with a frequency of $>1 \%$ were arbitrarily considered as highly expanded clones (HECs).

Results Both involved and uninvolved skin showed similar amounts of HECs at baseline $(14.3 \pm 4.2$ vs $15.0 \pm 0.0$ respectively; $\mathrm{p}=0.9$ ). However, at baseline BCR repertoires found in involved skin were clearly different from those in uninvolved skin; BCR clonal sequences were not shared between both compartments. Strikingly, RTX treatment led to a non-significant decrease of the number of HECs only in the involved skin $(14.3 \pm 4.2$ vs $9.0 \pm 4.2 ; p=0.22)$ before and after RTX respectively). The uninvolved skin did not show a decrease in HECs at all. Comparing the HECs in the involved skin before and after RTX, hardly any overlap could be detected between both time points. Similar results were found in the uninvolved skin samples.

Conclusions This high-resolution technique provides the first detailed characterisation of the BCR repertoire in SSc. The number of highly expanded clones (HECs) was not different when comparing involved versus uninvolved skin. 
This suggests that here is no BCR driven clonal expansion in involved skin. RTX treatment had a marginal effect on number of $B$ cell HECs in involved skin, but no effect on the number of HECs in uninvolved skin. 